\title{
Spatial versus object feature processing in human auditory cortex: a magnetoencephalographic study
}

\author{
Christoph S. Herrmann ${ }^{a, b, *}$, Daniel Senkowski ${ }^{a}$, \\ Burkhard Maess ${ }^{a}$, Angela D. Friederici ${ }^{\mathrm{a}}$ \\ ${ }^{a}$ Max-Planck-Institute of Cognitive Neuroscience, Postfach 500 355, 04303 Leipzig, Germany \\ ${ }^{b}$ Otto-von-Guericke Universität Magdeburg, 39016 Magdeburg, Germany
}

Received 31 July 2002; received in revised form 9 September 2002; accepted 12 September 2002

\begin{abstract}
The human visual system is divided into two pathways specialized for the processing of either objects or spatial locations. Neuroanatomical studies in monkeys have suggested that a similar specialization may also divide auditory cortex into two such pathways. We used the identical stimulus material in two experimental sessions in which subjects had to either identify auditory objects or their location. Magnetoencephalograms were recorded and M100 dipoles were fitted into individual brain models. In the right hemisphere, the processing of auditory spatial information lead to more lateral activations within the temporal plane while object identification lead to more medial activations. These findings suggest that the human auditory system processes object features and spatial features in distinct areas.

(C) 2002 Elsevier Science Ireland Ltd. All rights reserved.
\end{abstract}

Keywords: Auditory cortex; Dorsal; Human; Magnetoencephalogram; MEG; M100; N100m; Ventral

Magnocellular projections from the retina via the lateral geniculate nucleus (LGN) into primary visual cortex form a dorsal visual stream into parietal cortex and parvocellular projections form a ventral visual stream into temporal cortex. The ventral stream processes mainly object properties and projects to ventrolateral prefrontal cortex (VLPFC) while the dorsal stream processes mainly spatial locations and projects to dorsolateral prefrontal cortex (DLPFC) [20].

A similar division is also seen in the auditory cortex of monkeys. Within the lateral belt area of monkey auditory cortex, it has been shown that the anterior part projects to VLPFC while the posterior part projects to DLPFC [16]. Functionally this differentiation has been interpreted as a distinction between object and spatial processing in monkey auditory cortex [14]. Subsequent findings supported the notion of a functional specialization of the anterior part for types of monkey calls (object specialization) and of the posterior part for the spatial direction of monkey calls (spatial specialization) [19]. In humans, the middle frontal gyrus of DLPFC was activated bilaterally during auditory and visual spatial localization, indicating that auditory

\footnotetext{
* Corresponding author. Tel.: +49-341-9940-250; fax: +49 341 9940-204

E-mail address: herrmann@cns.mpg.de (C.S. Herrmann).
}

sound localization is processed in, or in the vicinity of, brain regions which are also involved in visual object localization [3]. Recently, distinct cortical pathways for either recognizing or localizing sounds have been reported for humans using functional magnetic resonance imaging (fMRI) [1,7]. However, so far no study has dissociated such a specialization within human auditory cortex using identical stimulus material for spatial and non-spatial tasks. Here, we present data which demonstrate that different parts of the human auditory cortex are activated as a function of different tasks either focusing attention on spatial or object features of the same stimuli as early as 120-160 ms after stimulus onset. The stimulus used in both tasks were identifiable sounds presented binaurally at seven different locations.

Five healthy male subjects participated in the study (ages ranging from 22 to 25 years, mean age 23 years). Complex auditory stimuli were presented binaurally from seven spatial locations. Prior to the experiment, each sound was presented via loudspeakers from each spatial location and recorded with a stereo head with microphones inside the ears (HEAD acoustics, HMS III.0). During the experiment, the sounds recorded with the stereo head were presented via air pressure headphones inside the magnetoencephalography (MEG) chamber. Individual hearing thresholds were 
determined for both ears of each subject and stimuli were presented $50 \mathrm{~dB}$ above. Each stimulus lasted $200 \mathrm{~ms}$. In an object task, subjects had to identify object targets (horn from a car) among six standard stimuli (ringing phone, digital chirp, whistle, kid's trumpet, bike horn, cuckoo clock) irrespective of their spatial location. In a spatial task, all stimuli presented from 30 degrees to the right had to be detected among the six other locations. The identical stimuli were used in both tasks. Subjects had to press a button with their right hand in response to targets and another button with their left hand in response to standards. All objects and locations were presented with equal probability. Each sound was presented 56 times, resulting in 336 trials for each standard condition which comprise either six objects or six locations. Only responses to standards were further evaluated, because of their higher signal-to-noise ratio and to avoid potential confounds with target detection. Mean values of reaction times and error rates were analyzed in an ANOVA with the factor task. Trials with reaction times exceeding 2.5 standard deviations from the mean were excluded.

MEG was recorded with a BTI 148 channel whole-head system (MAGNES WHS 2500). Horizontal and vertical electrooculogram was registered with four additional electroencephalograph electrodes. Data were sampled at 508.63 $\mathrm{Hz}$ (on-line $0.1 \mathrm{~Hz}$ analog high-pass and $100 \mathrm{~Hz}$ low-pass filtering) and digitally off-line filtered with a $20 \mathrm{~Hz}$ low-pass filter to reduce noise. Baselines were computed for each trial in the time interval $200 \mathrm{~ms}$ prior to stimulation and subtracted from the raw data before averaging. Averaging epochs lasted from $200 \mathrm{~ms}$ before to $900 \mathrm{~ms}$ after stimulus onset. Preprocessing of MEG data was carried out in a standard fashion [8]. Fig. 1 shows the event-related fields (ERFs) in response to standard stimuli in the object (blue) and spatial task (red). A clear M100 can be seen between 120 and $160 \mathrm{~ms}$. Dipoles were fitted for the individual averages of each subject using CURRY (Neuro Scan Labs, Sterling, VA). A realistically shaped boundary element model was used as volume conductor for each subject. For this purpose, models of each subject's brain were reconstructed from individual anatomical brain recordings such that a net of small triangles represents the outer surface of the brain. T1 weighted images from a 3 Tesla Bruker magnetic resonance imaging scanner were used for this purpose. One dipole was fitted into each hemisphere for the maximum of the global field power of each condition. After fitting, dipole locations were transformed into Talairach space [18] and superimposed onto the axial anatomical slice which best represented the location of the dipoles. Repeated-measures ANOVAs were carried out for the locations of the dipoles in Talairach space with factors (lateral/ medial), (anterior/posterior), (inferior/superior), hemisphere (left/right), and task (object/spatial).

The reaction times revealed no significant differences between the two tasks (object task: $501 \pm 70 \mathrm{~ms}$, spatial: $570 \pm 50 \mathrm{~ms}$ ) as indicated by an ANOVA. The ANOVA of the error rates yielded a significant effect of task $(F(1,4)=34.60, P<0.005)$, indicating fewer errors for the object task $(0.9 \pm 0.7 \%)$ than for the spatial task $(14.3 \pm 5.5 \%)$.

The dipole fits resulted in a quality of fit between $93 \%$ and $94 \%$ on average, reflecting a precise modeling of the data [13]. The ANOVA for the lateral/medial coordinate $(x)$ of the M100 dipoles yielded a significant interaction of hemisphere $x$ task $(F(1,4)=10.39, \quad P<0.05)$. Post-hoc analyses revealed that, within the right hemisphere, M100 dipoles were localized more lateral for the 'spatial' condition than for the 'object' condition $(F(1,4)=16.17$,
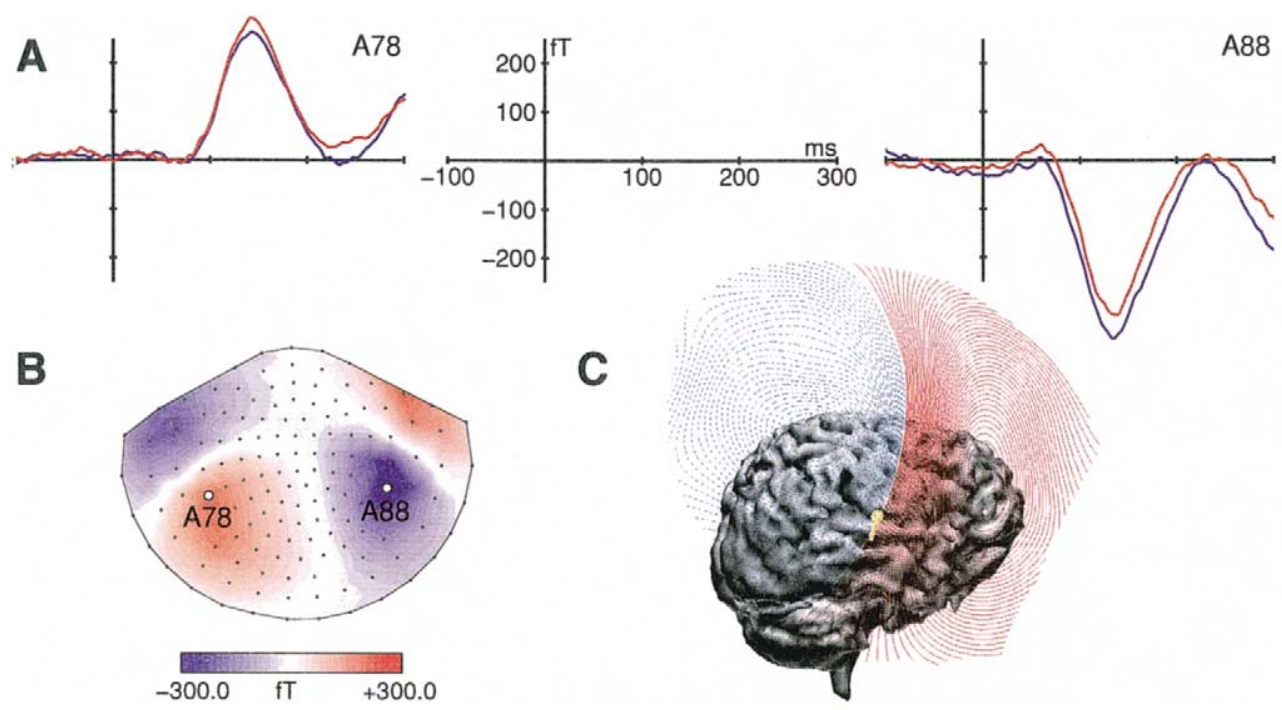

Fig. 1. (A) Averaged event-related fields for all five subjects over left (A78) and right (A88) auditory cortex for the object (blue) and the spatial (red) condition. (B) The location of the sensors is shown in the map which displays the topographical distribution of the object condition in the time interval 120-160 ms (top view, nose at top). (C) Magnetic fields and the fitted M100 dipole for the object task in the right hemisphere of an individual subject (side view, nose at right). 


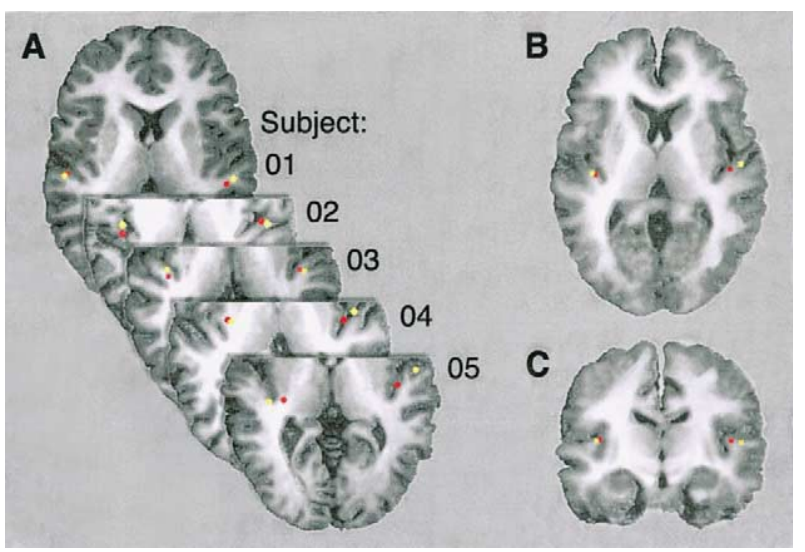

Fig. 2. M100 dipole locations for auditory object identification ('object' condition, red) and for localizing auditory objects in space ('spatial' condition, yellow). (A) Horizontal slices of the five individual brains. Horizontal (B) and coronal (C) slice of a mean brain computed from these five subjects. In the right hemisphere a clear separation of the two functional specializations can be seen: objects are processed more medially while space is processed more laterally.

$P<0.05, x($ spatial $)=47 \mathrm{~mm}, x($ object $)=41 \mathrm{~mm})$. Within the left hemisphere, differences of the lateral/medial coordinate $(x)$ of the M100 dipoles were not significant $(x($ spatial $)=-39 \mathrm{~mm}, \quad x($ object $)=-37 \mathrm{~mm})$. Fig. $2 \mathrm{~A}$ shows the specialization of the right hemisphere for spatial vs. object processing: M100 dipoles of all five subjects are mapped onto the individual MR slices of all five subjects for the mean $z$ coordinate of the dipoles: all dipoles of the 'spatial' condition (yellow) are more lateral than those of the 'object' condition (red) within the right hemisphere. Fig. $2 \mathrm{~B}$ shows the average dipole position mapped onto an averaged horizontal MR slice, Fig. 2C shows the averaged coronal slice. Both show a clear separation of the 'object' and 'spatial' dipoles within the right hemisphere.

The ANOVA of the anterior/posterior coordinate $(y)$ of the M100 dipoles yielded a significant main effect of task $(F(1,4)=10.86, P<0.05)$. Dipoles were located slightly more anterior for the 'spatial' condition $(y($ spatial $)=-16$ $\mathrm{mm})$ than for the 'object' condition $(y$ (object) $=-18 \mathrm{~mm})$. No effects were found for the factor inferior/superior $(z=10 \mathrm{~mm})$ or the dipole strengths.

Table 1 summarizes the mean dipole locations for both conditions and hemispheres. The localization of the M100 dipoles in Heschl's gyrus replicates earlier results which

Table 1

Talairach coordinates of mean dipole locations

\begin{tabular}{llrrr}
\hline Condition & Hemisphere & $x$ & $y$ & $z$ \\
\hline Object & Left & -37 & -20 & 11 \\
Spatial & Left & -39 & -19 & 9 \\
Object & Right & 41 & -17 & 9 \\
Spatial & Right & 47 & -14 & 10 \\
\hline
\end{tabular}

found M100 dipoles within the temporal plane, in Heschl's gyrus or slightly anterior or posterior $[9,11,17]$. The M100 probably represents the sum of activity in primary and nonprimary auditory areas.

Our results suggest that object features and spatial features are processed in different regions of human auditory cortex. The more lateral part processes predominantly spatial locations while the more medial part processes predominantly object information. This differentiation is more pronounced within the right hemisphere.

Reaction times did not differ significantly between tasks while higher error rates in the object task indicated that localizing one out of seven locations is somewhat more difficult than identifying one out of seven sounds. However, it seems unlikely that the observed different spatial activation of auditory cortex results from task difficulty. Varying the amount of attention or difficulty does influence the magnitude of fMRI responses in auditory cortex but not the location of the activation [6]. Even though fMRI activation need not behave in the same way as MEG dipoles this is an indication that attention did not confound our dipole localization.

The differential activation of auditory cortex for spatial and object tasks observed in the present study is taken to be related to different underlying neural mechanisms. It has been argued previously that auditory object identification relies upon frequency discrimination [19], while detecting the spatial source of sounds depends upon interaural time differences [4]. These interaural time differences are already computed in the superior olivary complex, but also in the inferior colliculi, the medial geniculate nucleus of the thalamus and auditory cortex processes this information with increasing sensitivity as compared to subcortical areas [4]. Among others, frequency properties of sounds are known to influence the auditory M100 [15]. Low frequencies map to more lateral and high frequencies to more medial areas within Heschl's gyrus [12]. Since sound source localization depends upon computation of interaural time differences of the low-frequency components (up to $2 \mathrm{KHz}$ ) [4] of the auditory signal, it makes sense that the 'spatial' dipole is located more laterally. Identification of sounds, however, requires the comparison of high frequencies, too, and thus leads to the more medial 'object' dipole. Of course, both regions might interact and fitting the data with just one dipole may be a simplification. The two different dipole locations might even represent two attentional foci within one tonotopic area rather than two completely separate streams.

The finding that the right hemisphere shows a clearer separation of the two pathways in our experiment is in line with the right-hemispheric dominance for processing the movement of auditory objects which is computed based on their location change [5]. While monkeys show a similar differentiation of 'object' and 'spatial' processing also in the left hemisphere [16], the human left hemisphere is specialized for language processing [2]. In the present 
study, the object stimulus items represent nameable auditory events which have been shown to activate language-related brain areas in the left hemisphere [10]. Their perception may therefore activate temporal cortex in the left hemisphere independently of the task. This would leave the preference for differential processing of object and spatial properties of sounds to the right hemisphere. Thus the observed right hemispheric dominance in differentiating object and spatial processing in human auditory cortex may be related to the human ability to process language in the left hemisphere.

We express our thanks to Yvonne Wolff and Kristin Schlosshauer who helped during data acquisition, to Erich Schröger who provided the stereo head, to Rudolf Rübsamen and Martin Meyer for valuable discussions of the data, and to Andrea Sandmann for the artwork. Daniel Senkowski was funded by the Deutsche Forschungsgemeinschaft (DFG, grant HE 3353/1). This study was supported by the Max Planck Society and the Leibniz Science Prize awarded to Angela Friederici by the DFG.

[1] Anourova, I., Nikouline, V.V., IImoniemi, R.J., Hotta, J., Aronen, H.J. and Carlson, S., Evidence for dissociation of spatial and nonspatial auditory information processing, Neuroimage, 14 (2001) 1268-1277.

[2] Binder, J.R., Frost, J.A., Hammeke, T.A., Cox, R.W., Rao, S.M. and Prieto, T., Human brain language areas identified by functional magnetic resonance imaging, J. Neurosci., 17 (1997) 353-362.

[3] Bushara, K.O., Weeks, R.A., Ishii, K., Catalan, M.-J., Tian, B., Rauschecker, J.P. and Hallett, M., Modality-specific frontal and parietal areas for auditory and visual spatial localization in humans, Nat. Neurosci., 2 (1999) 759-766.

[4] Fitzpatrick, D.C., Batra, R., Stanford, T.R. and Kuwada, S., A neuronal population code for sound localization, Nature, 388 (1997) 871-874.

[5] Griffiths, T.D., Rees, G., Rees, A., Green, G.G.R., Witton, C., Rowe, D., Büchel, C., Turner, R. and Frackowiak, R.S.J., Right parietal cortex is involved in the perception of sound movement in humans, Nat. Neurosci., 1 (1998) 74-79.

[6] Jäncke, L., Mirzazade, S. and Shan, N.J., Attention modulates activity in the primary and the secondary auditory cortex: a functional magnetic resonance imaging study in human subjects, Neurosci. Lett., 266 (1999) 125-128.
[7] Maeder, P.P., Meuli, R.A., Adriani, M., Bellmann, A., Fornari, E., Thiran, J.-P., Pittet, A. and Clarke, S., Distinct pathways involved in sound recognition and localization: a human fMRI study, Neuroimage, 14 (2001) 802-816.

[8] Maess, B., Koelsch, S., Gunter, T.C. and Friederici, A.D., Musical syntax is processed in Broca's area: an MEG study, Nat. Neurosci., 4 (2001) 540-545.

[9] Näätänen, R. and Picton, T., The N1 wave of the human electric and magnetic response to sound: a review and an analysis of the component structure, Psychophysiology, 24 (1987) 375-425.

[10] Opitz, B., Mecklinger, A. and Friederici, A.D., Functional asymmetry of human prefrontal cortex: encoding and retrieval of verbally and nonverbally coded information, Learn. Mem., 7 (2000) 58-96.

[11] Pantev, C., Bertrand, O., Eulitz, C., Verkind, C., Hampson, S., Schuierer, G. and Elbert, T., Specific tonotopic organizations of different areas of the human auditory cortex revealed by simultaneous magnetic and electric recordings, Electroenceph. clin. Neurophysiol., 94 (1995) 26-40.

[12] Pantev, C., Hoke, M., Lehnertz, K., Lückenhöner, B., Anogianakis, G. and Wittkowski, W., Tonotopic organization of the human auditory cortex revealed by transient auditory evoked magnetic fields, Electroenceph. clin. Neurophysiol., 69 (1988) 160-170.

[13] Picton, T.W., Alain, C., Woods, D.L., John, M.S., Scherg, M., Valdes-Sosa, P., Bosch-Bayard, J. and Trujillo, N.J., Intracerebral sources of human auditory-evoked potentials, Audiol. Neurootol., 4 (1999) 64-79.

[14] Rauschecker, J.P., Tian, B., Pons, T. and Mishkin, M., Serial and parallel processing in rhesus monkey auditory cortex, J. Comp. Neurol., 382 (1997) 89-103.

[15] Roberts, T.P.L. and Poeppel, D., Latency of auditory evoked M100 as a function of tone frequency, NeuroReport, 7 (1996) 1138-1140.

[16] Romanski, L.M., Tian, B., Fritz, J., Mishkin, M., GoldmanRakic, P.S. and Rauschecker, J.P., Dual streams of auditory afferents target multiple domains in the primate prefrontal cortex, Nature, 2 (1999) 1131-1136.

[17] Scherg, M. and von Cramon, D., Evoked dipole source potentials of the human auditory cortex, Electroenceph. clin. Neurophysiol., 65 (1986) 344-360.

[18] Talairach, J. and Tournoux, P., Co-planar Stereotaxic Atlas of the Human Brain, Thieme, New York, NY, 1988.

[19] Tian, B., Reser, D., Durham, A., Kustov, A. and Rauschecker, J.P., Functional specialization in rhesus monkey auditory cortex, Science, 292 (2001) 290-293.

[20] Ungerleider, L.G., Courtney, S.M. and Haxby, J.V., A neural system for human visual working memory, Proc. Natl. Acad. Sci. USA, 95 (1998) 883-890. 